\title{
On the EPR-chameleon experiment
}

\author{
Luigi AcCardi ${ }^{1}$, Kentaro Imafuku $^{2}$, Massimo Regoli $^{3}$ \\ Centro Vito Volterra, Università degli Studi di Roma "Tor Vergata" \\ WEB-page: http://volterra.mat.uniroma2.it,
}

\begin{abstract}
We construct a family of classical deterministic dynamical systems (triples formed by a state space, an initial distribution, a dynamics) parametrized by pairs of vectors $(a, b)$ in the unit circle in $\mathbb{R}^{2}$. The systems describe pairs of particles and the dynamics is strictly local, i.e. the dynamics $T_{a}^{(j)}$ of particle $j=1,2$ depends only on one of the two unit vectors, but not on the other. To each particle one associates a family of \pm 1 -valued observables $S_{a}^{(j)}$ $(j=1,2)$, also parametrized by vectors $a$ in the unit circle in $\mathbb{R}^{2}$. Moreover we assume that, if observable $S_{a}^{(j)}$ is measured on particle $j=1,2$, then the dynamics of this particle will be $T_{a}^{(j)}$ (chameleon effect).

Using these ingredients we prove that the dynamics and the initial distributions of the given systems can be chosen in such a way that, if the observable $S_{a_{j}}^{(j)}$ is measured on particle $j=1,2$, then the EPR correlations $-\cos \left(a_{1}-a_{2}\right)$ are reproduced.

This theoretical construction is then used to realize the following experiment: a central computer $S$ (source) sends the same signal to two local computers $A$ and $B$. After receiving the signal $A$ (resp. $B$ ) chooses one vector $a$ (resp. $b$ ) in the unit circle in $\mathbb{R}^{2}$ and computes the value of the corresponding observable $S_{a}^{(1)}$ (resp. $S_{b}^{(2)}$ ). These values are uniquely defined by the deterministic dynamics and the choices are independent of one-another.

The local computers send back to the central one the results of the evaluation of these \pm 1 -valued functions and the central computer evaluates the empirical correlations according to the usual statistical rules.

As a result the EPR correlations are reproduced with very good approximation and the Bell inequality is violated by a classical, macroscopic deterministic system performing purely local choices.
\end{abstract}

The program to run the experiment is available from the WEB-page: http://volterra.mat.uniroma2.it.

\section{Physical interpretation of the experiment}

The present paper extends and clarifies the result of the EPR-chameleon experiment, proposed in [?, ?]. The goal of the experiment is (i) to construct a classical, deterministic, reversible, dynamical system which reproduces the EPR correlations by local choices; (ii) to use this model to give an experimental proof of the fact that macroscopic systems with the above mentioned characteristics can violate Bell's inequalities for principle and not for contingent reasons.

\footnotetext{
${ }^{1}$ accardi@volterra.mat.uniroma2.it

2imafuku@volterra.mat.uniroma2.it

${ }^{3}$ massimo@volterra.mat.uniroma2.it
} 
We do not pretend our model to be a hidden variable model for the EPR experiments.

Our goal is to prove that Bell's statement [?]: "... the statistical predictions of quantum mechanics are incompatible with local predetermination ..." is theoretically and experimentally unjustified if by "statistical predictions of quantum mechanics" we mean the EPR correlations and by "local predetermination" the possibility of reproducing these correlations by a classical deterministic macroscopic system subject to local choices.

The main hidden mathematical assumption in Bell's argument was pointed out in [?] (cf. [?] for a survey) where the nonkolmogorovian character of the EPR correlations was first noticed and the crucial role played by conditional probability emphasized.

In [?] (cf. also [?, ?]) von Neumann's measurement theory was extended to include in it the two basic conditions of locality and causality and the physical principle which allows to exploit Bell's hidden mathematical assumption was individuated in the "chameleon effect": the dynamics of a system may depend on the observables we want to measure (or, more generally, on the local environment).

For such systems what you measure is a response to an interaction and therefore, when dealing with them, one should not speak of platonic (i.e. in principle unobservable) things such as "values of non measured observables", but rather of "instruction kits telling them what to do when meeting a measurement apparatus" [?]. This is opposed to ballot box (or Einstein) reality in which you measure what was there (independently of the environment).

More recently the main idea of the chameleon effect, i.e. that the local interaction with the apparatus may have observable effects on the global statistics, has been used to construct models related to the "detection loophole" [?, ?, ?, ?] (cf. [?, ?, ?, ?, ?] for earlier discussions). In these papers one constructs local hidden variable models in which, by assuming a partial inefficiency of the detectors, one can riproduce the EPR correlations (or similar).

On the contrary, the dynamical theory of [?] is based on first principles, and does not introduce contingent limitations of efficiency: even postulating 100\% efficiency of all the instruments involved, because of the chameleon effect, the local deterministic dynamics of the two particles are different and one can use this freedom to construct examples of local deterministic classical dynamical systems as specified by:

- a state space (hidden parameters)

- a deterministic dynamics (chameleon effect)

- an initial probability measure (preparation of the experiment)

whose statistics is non classical.

The present paper produces a concrete example of such a system, which reproduces the EPR correlations hence violates Bell's inequality. Moreover we give an experimental proof of its local simulability.

The conceptual and experimental differences between the present approach and the detection loophole are discussed in Section (??) below. Beyond the physical considerations, discussed in this section, there is also a mathematical argument that proves the disjoint nature of the two topics concerning coincidences and efficiency of the detectors. In all papers involving efficiencies of detectors, these are explicitly built into the model. On the contrary, as anybody can verify looking at Section (??) below, in our mathematical model neither inefficiencies nor coincidences play any role. We have only a deterministic dynamics and an initial probability distribution. It is only at the simulation level that the natural interpretation of the local factors as conditional probabilities over coincidences emerges. This is because a factor $2 \pi$, appearing in a change of variables both in numerator and denominator is simplified in the calculations but must not be simplified in order to allow local simulation (cf. Section (2), (3), (4)). 
Our experiment describes the following classical dynamical system. A source $C$ (central computer) produces pairs of particles $\left(S_{1}, S_{2}\right)$ which travel in different directions and after some time, each particle interacts with a measurement apparatus $\left(M_{1}, M_{2}\right)$. By the chameleon effect the dynamical evolution of each particle depends on the setting of the nearby apparatus, but not on the setting of the apparatus interacting with the other particle (locality).

Even if, as just stated, our model has not the pretense to mimick the real singlet experiments, an analogy with them will be useful for the intuition of what is going on in it. In this analogy one can interpret $\left(S_{1}, S_{2}\right)$ as a pair of photons and $M_{1}, M_{2}$ as polarizers. The detector and the coincidence counters as well as the space-time trajectories of the two particles are not explicitly modeled in our dynamical system.

Following the standard prescriptions of (von Neumann) measurement theory, we model the joint evolution of the system $S=\left(S_{1}, S_{2}\right)$ (i.e. the two particles) and the apparatus $M=$ $\left(M_{1}, M_{2}\right)$ (i.e. the two measurement apparata). We want to incorporate in von Neumann's scheme of measurement the two requirements of locality and causality. The local structure of our dynamical system will be reflected in the model through:

(i) a local structure of the initial state (probability measure) of the composite $(S, M)$ system (cf. (??))

(ii) a local structure of the dynamics of the sub-systems $\left(S_{1}, M_{1}\right)$ (particle 1, apparatus 1), $\left(S_{2}, M_{2}\right)$ (cf. (??), (??), (??)).

We formulate these locality conditions only in the classical case and for our specific model, but there is no difficulty in rephrasing them so to include the general (classical and quantum) case.

We assume that, at the moment of emission from the source, the two particles are in the same (microscopic) state (cf. (??)) and that the state of the apparatus is not changed by the interaction with the particle. For example, if the polarizer was oriented in direction $a$ before interacting with the photon, the same will be true after interaction (cf. (??), (??)).

The causality condition is reflected in the fact that the initial state of $\left(S_{1}, S_{2}\right)$ does not depend on the settings of the apparata $M_{1}, M_{2}$ : in fact, at the time of emisison from the source, the particles cannot know which measurements will be done on them.

We assume moreover that the dynamics of the two sub-systems $\left(S_{1}, M_{1}\right),\left(S_{2}, M_{2}\right)$ are independent, i.e. either sub-system does not feel the influence of the other one. This is the locality condition as formulated by EPR, Bell,... . We discretize time and consider only the initial and final time of the experiment.

To each particle $S_{1}, S_{2}$ we associate a set of observables

$$
\left\{S_{a}^{(1)}: a \in[0,2 \pi\}\right\} ; \quad\left\{S_{b}^{(2)}: b \in[0,2 \pi]\right\}
$$

These observables are modeled by functions defined on a space $\Omega$ and with values +1 or -1 .

In our model just as for photons, the actual detection takes place after interaction with the polarizers. Thus, if experimenters 1, 2 want to measure $S_{a}^{(1)}, S_{b}^{(2)}$ respectively then they will prepare the polarizer (or magnet) 1, 2 oriented in direction $a$ (resp. b). In other words the initial state of the polarizer depends on the observable we want to measure. Since the particle interacts with the polarizer, the same will be true for its dynamics. In other words: the chameleon effect is a natural consequence of standard measurement theory.

Another consequence of what just said is that the state of the composite system $\left(S_{1}, S_{2}\right.$, $M_{1}, M_{2}$ ) will depend on the pair of measurements $a, b$. This is the known contextuality 
requirement but, as shown in section (??), by itself this is by far not sufficient to rule out the validity of Bell's inequality: for this a more subtle analysis is required.

Because of the different dynamics (chameleon effect) the two particles have different trajectories hence even if they leave the source at the same time, they do not necessarily interact simultaneously with the corresponding apparatus. When they do we say that a coincidence takes place. In agreement with what is done in all the EPR type experiments also in our experiment the statistical countings are conditioned on coincidences because the correlations are equal time correlations: this also assures that only photons coming from entangled pairs are considered in the correlations. Since the measurement apparata are approximatively equidistant from the source, if the time interval between the emission of two consecutive pairs is much larger than the coincidence interval (i.e. the time interval within which two events are considered as simultaneous) then, assuming $100 \%$ efficiency of the detectors and of the clocks (what we will always do in the present paper), we can be sure that only particles belonging to the same pair can give rise to coincidences.

In EPR type experiments coincidences are measured either with very precise clocks (coincidence intervals of order of nanoseconds) as in [?] or by direct connection of the polarizers to a coincidence counter, as in [?]. The former technique allows a larger space separation among the polarizers (a feature which is relevant for quantum cryptography); the latter, as advocated in [?], improves precision in the coincidence counting.

If the initial state of the two particles is chosen at random, also the number of coincidences will be random. Assuming reasonable ergodic properties of the system, we can expect that this number will have small fluctuations around its mean value.

In our model this mean value is independent of any special choice, in particular it does not depend on the setting of the far away apparata. Also this fact is in agreement with the experimental fact that: the size of the selected sample is found constant [?].

\section{A local, deterministic, reversible, classical dy- namical system realizing the EPR correlation}

In this section we describe the mathematical model on which the computer experiment is based. According to the general description of the chameleon effect [?, ?] we need an initial probability measure and a dynamics. We construct these objects in the present section and we will discuss their interpretation in the following sections.

Define, for $\sigma_{1}, \sigma_{1} \in[0,2 \pi]$, the functions

$$
T_{1, a}^{\prime}\left(\sigma_{1}\right)=\frac{\sqrt{2 \pi}}{4}\left|\cos \left(\sigma_{1}-a\right)\right|, \quad T_{2, b}^{\prime}\left(\sigma_{2}\right)=\sqrt{2 \pi}
$$

and define the maps (dynamics)

$$
\begin{aligned}
& T_{1, a}\left(p_{1}\right)=\left(s_{1, a}\left(p_{1}\right), m_{1, a}\left(p_{1}\right)\right) \quad, \quad T_{2, b}\left(p_{2}\right)=\left(s_{2, b}\left(p_{2}\right), m_{2, b}\left(p_{2}\right)\right) \\
& p_{1}=\left(\sigma_{1}, \lambda_{1}\right) ; \quad s_{1, a}\left(\sigma_{1}, \lambda_{1}\right)=\sigma_{1}, \quad m_{1, a}\left(\sigma_{1}, \lambda_{1}\right)=\lambda_{1} \frac{1}{T_{1, a}^{\prime}\left(\sigma_{1}\right)} \\
& p_{2}=\left(\sigma_{2}, \lambda_{2}\right) ; \quad s_{2, b}\left(\sigma_{2}, \lambda_{2}\right)=\sigma_{2}, \quad m_{2, b}\left(\sigma_{2}, \lambda_{2}\right)=\lambda_{2} \frac{1}{T_{2, b}^{\prime}\left(\sigma_{2}\right)}
\end{aligned}
$$


Define moreover the measures $\left(\sigma_{1}, \lambda_{1}, \sigma_{2}, \lambda_{2} \in[0,2 \pi]\right)$ :

$$
\begin{gathered}
p_{S}\left(\sigma_{1}, \sigma_{2}\right)=\frac{1}{2 \pi} \delta\left(\sigma_{1}-\sigma_{2}\right) d \sigma_{1} d \sigma_{2} \\
p_{1, a}\left(\sigma_{1}, \lambda_{1}\right)=\delta\left(m_{1, a}\left(\sigma_{1}, \lambda_{1}\right)-m_{a}\right) d \lambda_{1} \\
p_{2, b}\left(\sigma_{2}, \lambda_{2}\right)=\delta\left(m_{2, b}\left(\sigma_{2}, \lambda_{2}\right)-m_{b}\right) d \lambda_{2}
\end{gathered}
$$

Lemma 1 The measure

$$
p_{S}\left(\sigma_{1}, \sigma_{2}\right) p_{1, a}\left(\sigma_{1}, \lambda_{1}\right) p_{2, b}\left(\sigma_{2}, \lambda_{2}\right) d \sigma_{1} \sigma_{2} d \lambda_{1} d \lambda_{2}
$$

is a probability measure on $[0,2 \pi]^{4}$.

Proof. The positivity is obvious. The normalization condition

$$
\int p_{S}\left(\sigma_{1}, \sigma_{2}\right) p_{1, a}\left(\sigma_{2}, \lambda_{1}\right) p_{2, b}\left(\sigma_{2}, \lambda_{2}\right) d \sigma_{1} \sigma_{2} d \lambda_{1} d \lambda_{2}=1
$$

follows from a simple calculation (cf. also the proof of Lemma (??)).

Remark 1 It would be tempting to interpret the measures (??), (??) as conditional distributions of the state of the apparatus given the incoming state of the correponding photon. However this is not possible because it happens that:

$$
\int p_{1, a}\left(\sigma_{1}, \lambda_{1}\right) d \lambda_{1}=\frac{\sqrt{2 \pi}}{4}\left|\cos \left(\sigma_{1}-a\right)\right| \neq 1, \quad \int p_{2, b}\left(\sigma_{2}, \lambda_{2}\right) d \lambda_{2}=\sqrt{2 \pi} \neq 1
$$

As shown in section (??) the inequalities in (??) are necessary for the violation of Bell's inequality.

Lemma 2 Let the \pm 1 -valued maps $S_{a}^{(1)}\left(\sigma_{1}, \mu_{1}\right), S_{x}^{(2)}\left(\sigma_{2}, \mu_{2}\right)\left(\sigma_{j}, \mu_{j} \in[0,2 \pi]\right)$ be given by

$$
S_{a}^{(1)}(\sigma, \mu)=\operatorname{sgn}(\cos (\sigma-a)) \quad, \quad S_{x}^{(2)}=-S_{x}^{(1)}
$$

then, in the above notations,

$$
\begin{aligned}
& \int S_{1}^{(a)}\left(s_{1}\left(\sigma_{1}, \lambda_{1}\right), m_{1}\left(\sigma_{1}, \lambda_{1}\right)\right) S_{2}^{(b)}\left(s_{2}\left(\sigma_{2}, \lambda_{2}\right), m_{2}\left(\sigma_{2}, \lambda_{2}\right)\right) \\
& \times p_{S}\left(\sigma_{1}, \sigma_{2}\right) p_{1, a}\left(\sigma_{1}, \lambda_{1}\right) p_{2, b}\left(\sigma_{2}, \lambda_{2}\right) d \sigma_{1} d \sigma_{2} d \lambda_{1} d \lambda_{2}=-\cos (a-b)
\end{aligned}
$$

Proof. With the choices (??), (??), (??), the correlations (??) become

$$
\begin{gathered}
\iiint \int S_{a}^{(1)}\left(s_{1, a}\left(\sigma_{1}, \lambda_{1}\right), m_{1, a}\left(\sigma_{1}, \lambda_{1}\right)\right) S_{b}^{(2)}\left(s_{2, b}\left(\sigma_{2}, \lambda_{2}\right), m_{2, b}\left(\sigma_{2}, \lambda_{2}\right)\right) \\
\delta\left(m_{1, a}\left(\sigma_{1}, \lambda_{1}\right)-m_{a}\right) \delta\left(m_{2, b}\left(\sigma_{2}, \lambda_{2}\right)-m_{b}\right) p_{S}\left(\sigma_{1}, \sigma_{2}\right) d \lambda_{1} d \lambda_{2} d \sigma_{1} d \sigma_{2}
\end{gathered}
$$

Changing variables

$$
\begin{gathered}
m_{1, a}\left(\sigma_{1}, \lambda_{1}\right)=\mu_{1} \\
m_{2, b}\left(\sigma_{2}, \lambda_{2}\right)=\mu_{2} \\
m_{1, a}^{\prime}\left(\sigma_{1}, \lambda_{1}\right) d \lambda_{1}=d \mu_{1}
\end{gathered}
$$




$$
m_{2, b}^{\prime}\left(\sigma_{2}, \lambda_{2}\right) d \lambda_{2}=d \mu_{2}
$$

and noting that for almost all $a, b, \sigma_{1}, \sigma_{2} \in[0,2 \pi]$ the functions

$$
m_{1, a}\left(\sigma_{1}, \cdot\right), m_{2, b}\left(\sigma_{2}, \cdot\right):[0,2 \pi] \rightarrow[0,2 \pi]
$$

are invertible, one has from (??), (??), (??)

$$
\begin{aligned}
& d \lambda_{1}=\frac{1}{m_{1, a}^{\prime}\left(\sigma_{1}, m_{1, a}^{-1}\left(\sigma_{1}, \mu_{1}\right)\right)} d \mu_{1}=: \tilde{T}_{1, a}^{\prime}\left(\sigma_{1}, \mu_{1}\right) d \mu_{1} \\
& d \lambda_{2}=\frac{1}{m_{2, b}^{\prime}\left(\sigma_{2}, m_{2, b}^{-1}\left(\sigma_{2}, \mu_{2}\right)\right)} d \mu_{2}=: \tilde{T}_{2, b}^{\prime}\left(\sigma_{2}, \mu_{2}\right) d \mu_{2}
\end{aligned}
$$

and, after the change of variables, (??) becomes

$$
\begin{gathered}
\iiint \int S_{a}^{(1)}\left(s_{1, a}\left(\sigma_{1}, m_{1, a}^{-1}\left(\sigma_{1}, \mu_{1}\right)\right), \mu_{1}\right) S_{b}^{(2)}\left(s_{2, b}\left(\sigma_{2}, m_{2, b}^{-1}\left(\sigma_{2}, \mu_{2}\right)\right), \mu_{2}\right) \\
\tilde{T}_{1, a}^{\prime}\left(\sigma_{1}, \mu_{1}\right) \tilde{T}_{2, b}^{\prime}\left(\sigma_{2}, \mu_{2}\right) \delta\left(\mu_{1}-m_{a}\right) \delta\left(\mu_{2}-m_{b}\right) p_{S}\left(\sigma_{1}, \sigma_{2}\right) d \mu_{1} d \mu_{2} d \sigma_{1} d \sigma_{2}
\end{gathered}
$$

Because of our choice of the functions $S_{a}^{(1)}, S_{b}^{(2)}, T_{1, a}^{\prime}, T_{2, b}^{\prime}$, these have the form

$$
\begin{aligned}
S_{a}^{(1)}\left(\sigma_{1}\right) & :=S_{a}^{(1)}\left(s_{1, a}\left(\sigma_{1}, m_{1, a}^{-1}\left(\sigma_{1}, m_{a}\right), \mu_{1}\right)\right. \\
S_{b}^{(2)}\left(\sigma_{2}\right) & :=S_{b}^{(2)}\left(s_{2, b}\left(\sigma_{2}, m_{2, b}^{-1}\left(\sigma_{2}, m_{b}\right), \mu_{2}\right)\right. \\
& T_{1, a}^{\prime}\left(\sigma_{1}\right):=\tilde{T}_{1, a}^{\prime}\left(\sigma_{1}, m_{a}\right) \\
& T_{2, b}^{\prime}\left(\sigma_{2}\right):=\tilde{T}_{2, b}^{\prime}\left(\sigma_{2}, m_{b}\right)
\end{aligned}
$$

in the sense that the right hand side depends only on the variables written on the left hand side. Therefore (??) becomes

$$
\iint S_{a}^{(1)}\left(\sigma_{1}\right) S_{b}^{(2)}\left(\sigma_{2}\right) T_{1, a}^{\prime}\left(\sigma_{1}\right) T_{1, b}^{\prime}\left(\sigma_{2}\right) p_{S}\left(\sigma_{1}, \sigma_{2}\right) d \sigma_{1} d \sigma_{2}
$$

Finally, since

$$
p_{S}\left(\sigma_{1}, \sigma_{2}\right)=\frac{1}{2 \pi} \delta\left(\sigma_{1}-\sigma_{2}\right)
$$

we arrive at

$$
\int S_{a}^{(1)}(\sigma) S_{b}^{(2)}(\sigma) T_{1, a}^{\prime}(\sigma) T_{1, b}^{\prime}(\sigma) \frac{d \sigma}{2 \pi}=-\int_{0}^{2 \pi} \cos (\sigma-b) \operatorname{sgn}(\cos (\sigma-a)) d \sigma=-\cos (b-a)
$$

which is the thesis.

Remark 2 Given (??) the expression (??), (??) is precisely the one which was taken as starting point in the paper [?]. The result of the present section proves that the interpretation in terms of reduced dynamics proposed in that paper was correct: the Heisenberg dynamics used in [?] is indeed the reduced dynamics of the reversible dynamics considered in the present paper. 


\section{The simulation problem}

Starting from this section until the end of Section (??) we describe two methods of local simulation of the deterministic model described in Section (??). The method we actually used in the realization of our experiment, is the one described in Section (??). The other method corresponds to previous versions of the experiment [?] (this method corresponds to the option "old" in the menu of the programme in the web) and is interesting because it illustrates the difference between the theoretical problem, solved in Section 2, and the actual simulation method.

In order to simulate locally the probability measure (??) we normalize the local factors at $a$ and $b$, obtaining the following expression for the correlations (??)

$$
\begin{gathered}
\int_{0}^{2 \pi} \int_{0}^{2 \pi} \frac{d \sigma_{1} d \sigma_{2}}{2 \pi} \delta\left(\sigma_{1}-\sigma_{2}\right) \frac{2 \pi}{4}\left|\cos \left(\sigma_{1}-a\right)\right| \\
{\left[\int_{0}^{2 \pi} S_{a}^{(1)}\left(\sigma_{1}, \lambda_{1}\right) \frac{p_{1, a}\left(\sigma_{1}, \lambda_{1}\right) d \lambda_{1}}{Z_{a}^{(1)}\left(\sigma_{1}\right)}\right]\left[\int_{0}^{2 \pi} S_{b}^{(2)}\left(\sigma_{2}, \lambda_{2}\right) \frac{p_{2, b}\left(\sigma_{2}, \lambda_{2}\right) d \lambda_{2}}{Z_{b}^{(2)}\left(\sigma_{2}\right)}\right]}
\end{gathered}
$$

Integrating the $\delta$-function, simplifying the $2 \pi$-factor and introducing the notations

$$
\tilde{p}_{j, x}\left(\sigma_{j}, \lambda_{j}\right) d \lambda_{j}=\frac{p_{j, x}\left(\sigma_{j}, \lambda_{j}\right)}{Z_{x}^{(j)}\left(\sigma_{j}\right)} d \lambda_{j} \quad ; \quad j=1,2 ; \quad x=a, b
$$

$\left(Z_{a}^{(j)}\left(\sigma_{j}\right)\right.$ being the normalization factor) we obtain

$$
\int_{0}^{2 \pi} d \sigma\left[\int_{0}^{2 \pi} \frac{|\cos (\sigma-a)|}{4} S_{a}^{(1)}\left(\sigma, \lambda_{1}\right) \tilde{p}_{1, a}\left(\sigma, \lambda_{1}\right) d \lambda_{1}\right]\left[\int_{0}^{2 \pi} S_{b}^{(2)}\left(\sigma, \lambda_{2}\right) \tilde{p}_{2, b}\left(\sigma, \lambda_{2}\right) d \lambda_{2}\right]
$$

Given our choice (??) of $S_{a}^{(1)}, S_{b}^{(2)}$, the second integral in square brackets is equal to $S_{b}^{(2)}(\sigma)$. This is evaluated locally by computer 2 . The integrand of the first integral in square brackets is equal to $\frac{1}{4} \cos (\sigma-a)$. In order to simulate locally this integral we replace in it the function $\frac{1}{4}|\cos (\sigma-a)| S_{a}^{(1)}\left(\sigma, \lambda_{1}\right)$ by any function $\hat{S}_{a}^{(1)}(\sigma, \lambda)$, with values \pm 1 , such that

$$
\int_{0}^{2 \pi} \hat{S}_{a}^{(1)}(\sigma, \lambda) \tilde{p}_{1, a}\left(\sigma, \lambda_{1}\right) d \lambda_{1}=\frac{1}{4} \cos (\sigma-a)
$$

Such function is easily constructed because $\tilde{p}_{1, a}\left(\sigma, \lambda_{1}\right) d \lambda_{1}$ is a probability measure. This integral is evaluated locally by computer 1 . After this, and with the notation

$$
\hat{S}_{b}^{(2)}(\sigma, \lambda)=S_{b}^{(2)}(\sigma, \lambda)
$$

we arrive to integral

$$
\begin{gathered}
\int_{0}^{2 \pi} d \sigma\left[\int_{0}^{2 \pi} \hat{S}_{a}^{(1)}\left(\sigma, \lambda_{1}\right) \tilde{p}_{1, b}\left(\sigma, \lambda_{1}\right) d \lambda_{1}\right]\left[\int_{0}^{2 \pi} \hat{S}_{b}^{(2)}\left(\sigma, \lambda_{2}\right) \tilde{p}_{2, b}\left(\sigma, \lambda_{2}\right) d \lambda_{2}\right] \\
=\int_{0}^{2 \pi} d \sigma \frac{\cos (\sigma-a)}{4}(-\operatorname{sgn}(\cos (\sigma-b)))
\end{gathered}
$$

To simulate this integral directly would be easy (this is what was done in the first experiment [?]). However this would require that the integrals in $d \lambda_{1}, d \lambda_{2}$ should be calculated locally 
by computer 1 and 2 respectively and only the two integrals in square brackets would be sent to the central computer. If, in greater coherence with the experiments, we want the local computers to send to the central computer only sequences of \pm 1 (i.e., the values of $\left.\hat{S}_{a}^{(1)}\left(\sigma, \lambda_{1}\right), \hat{S}_{b}^{(2)}\left(\sigma, \lambda_{2}\right)\right)$, then we have to generate a sequence of $\left(\sigma_{j}\right)$ in $[0,2 \pi]$ which realizes this goal. To this purpose, following the standard Monte Carlo procedure, we normalize the $d \sigma$-integral by dividing and multiplying by $2 \pi$

$$
2 \pi\left[\int_{0}^{2 \pi} \frac{d \sigma}{2 \pi} \frac{\cos (\sigma-a)}{4}(-\operatorname{sgn}(\cos (\sigma-b)))\right]
$$

this leads to the integral

$$
2 \pi\left\{\int_{0}^{2 \pi} \frac{d \sigma}{2 \pi}\left[\int_{0}^{2 \pi} \hat{S}_{a}^{(1)}\left(\sigma, \lambda_{1}\right) \tilde{p}_{1, a}\left(\sigma, \lambda_{1}\right) d \lambda_{1}\right]\left[\int_{0}^{2 \pi} \hat{S}_{b}^{(2)}\left(\sigma, \lambda_{2}\right) \tilde{p}_{2, b}\left(\sigma, \lambda_{2}\right) d \lambda_{2}\right]\right\}
$$

in which one can now easily approximate locally each piece with Riemann sums

$$
\frac{2 \pi}{N} \sum_{\sigma_{j}}\left[\frac{1}{K_{1}} \sum_{k_{1}} \hat{S}_{a}^{(1)}\left(\sigma_{j}, \lambda_{a, k_{1}}^{(j)}\right)\right]\left[\frac{1}{K_{2}} \sum_{k_{2}} \hat{S}_{b}^{(2)}\left(\sigma_{j}, \lambda_{b, k_{2}}^{(j)}\right)\right]
$$

This gives the following prescriptions: let

$C$ denote central computer

$A$ Experimenter 1

$B$ Experimenter 2

1. $C$ produces any sequence $\sigma_{j}$ in $[0,2 \pi]$ distributed according to $\frac{d \sigma}{2 \pi}$. It is not necessary that the sequence $\left(\sigma_{j}\right)$ has good chaotic properties: any equidistributed sequence in $[0,2 \pi]$ can be used.

2. For each $\sigma_{j}, A$ produces $\left(\lambda_{a, k_{1}}^{(j)}\right)_{k_{1}}$ distributed according to $\hat{p}_{1, a}(d \lambda)$ and produces the sequence of values $\hat{S}_{a}^{(1)}\left(\sigma_{j}, \lambda_{a, k_{1}}^{(j)}\right) \in\{ \pm 1\}$

3. For each $\sigma_{j}, B$ produces $\left(\lambda_{b, k_{2}}^{(j)}\right)_{k_{2}}$ distributed according to $\hat{p}_{2, b}(d \lambda)$ and produces the sequence of values $\hat{S}_{b}^{(2)}\left(\sigma_{j}, \lambda_{b, k_{2}}^{(j)}\right) \in\{ \pm 1\}$.

4. $A$ and $B$ send these two sequences to $C$

5. $C$ takes the arithmetic mean of the product of the two sequences and multiplies the result by $2 \pi$ thus compensating the division by $2 \pi$, introduced in Step (1).

Remark 3 The above simulation procedure has two unsatisfactory features: (i) the artificial (although standard in Monte Carlo simulations) "multiplication and division by $2 \pi$ "; (ii) the fact that, before multiplication by $2 \pi$, the correlations (??) for $a=b$ are not equal to -1 but to $-1 / 2 \pi$, hence the singlet condition $S_{a}^{(1)}=-S_{b}^{(2)}$, although globally satisfied due to Lemma (2), is not satisfied at each step of the simulation procedure.

In Section (5) below we describe a simulation method which is free from these drawbacks. Before that, in the following section, we describe the probabilistic origins of this difficulty. 


\section{Probabilistic interpretation of the multiplication and division by $2 \pi$}

In this section we propose a probablistic interpretation of the decomposition (??) before simplification of the two $2 \pi$ factors. This interpretation gives the key for the simulation used in Section (??).

Let us first discuss, in some generality, which kind of properties it is reasonable to expect from a "locally simulable" measure, i.e. a measure that, in addition of having the local structure (??) is also such that it can be split into 3 pieces each of which is simulable in a different computer.

Recall that in real experiments the countings are conditioned on coincidences and that these occur at the polarizers site. By locality we can expect, for a locally simulable probability measure a structure of the form

$$
p_{S}\left(\sigma_{1}, \sigma_{2}\right) E_{\Gamma_{c}}(\cdot)\left(\sigma_{1}, \sigma_{2}\right) d \sigma_{1} d \sigma_{2}
$$

where $p_{S}$ is the distribution at the source and $E_{\Gamma_{c}}(\cdot)\left(\sigma_{1}, \sigma_{2}\right)$ denotes the conditional distribution of the apparatus variables given simultaneous interaction with particles $S_{1}, S_{2}$ in the state $\sigma_{1}, \sigma_{2}$ respectively. Denoting $\Gamma_{c}$ the event: "a coincidence occurs at the time of measurement", the conditional expectation $E_{\Gamma_{c}}$ can be written as

$$
E_{\Gamma_{c}}(F)=\frac{E\left(F \chi_{\Gamma_{c}}\right)}{P\left(\Gamma_{c}\right)}
$$

where $F$ is any function, $\chi_{\Gamma_{c}}(\omega)=1$ if $\omega \in \Gamma_{c}$ and $=0$ if $\omega \notin \Gamma_{c}$ and $P\left(\Gamma_{c}\right)$ is the probability of $\Gamma_{c}$.

Moreover it is reasonable to expect that, given coincidence and the pair $\left(\sigma_{1}, \sigma_{2}\right)$, the results of measurement at instruments 1 and 2 should be independent events: in fact, once given $\Gamma_{c}, \sigma_{1}, \sigma_{2}$, what happens at 1 (resp. 2) only depends on the local apparatus variable $\lambda_{1}$ (resp. $\lambda_{2}$ ). Thus, if $F_{1, a}, F_{2, b}$ are local observables (local in the sense that $\left.F_{j, x}\left(\sigma_{1}, \sigma_{2}, \lambda_{1}, \lambda_{2}\right)=F_{j, x}\left(\sigma_{j}, \lambda_{j}\right)(j=1,2, x=a, b)\right)$, one should have

$$
E\left(F_{1, a} F_{2, b} \chi_{\Gamma_{c}}\right)\left(\sigma_{1}, \sigma_{2}\right)=E\left(F_{1, a} \chi_{\Gamma_{c}}\right)\left(\sigma_{1}, \sigma_{2}\right) E\left(F_{2, b} \chi_{\Gamma_{c}}\right)\left(\sigma_{1}, \sigma_{2}\right)
$$

Our model is Section (2) also satisfies the additional condition

$$
E\left(F_{j, x} \chi_{\Gamma_{c}}\right)\left(\sigma_{1}, \sigma_{2}\right)=E\left(F_{j, x} \chi_{\Gamma_{c}}\right)\left(\sigma_{j}\right) ; j=1,2
$$

but a model not satisfying this condition would not violate locality because $\left(\sigma_{1}, \sigma_{2}\right)$ is the state at the source, where the 2 particles may be very near each other.

In any case, assuming both the above conditions, we would find for the correlations

$$
\begin{gathered}
\left\langle S_{a}^{(1)} S_{b}^{(2)}\right\rangle=\iint p_{S}\left(\sigma_{1}, \sigma_{2}\right) d \sigma_{1} d \sigma_{2} E_{\Gamma_{c}}\left(S_{a}^{(1)} S_{b}^{(2)}\right)\left(\sigma_{1}, \sigma_{2}\right) \\
=\frac{1}{P\left(\Gamma_{c}\right)} \iint p_{S}\left(\sigma_{1}, \sigma_{2}\right) d \sigma_{1} d \sigma_{2} E_{1, a}\left(S_{a}^{(1)} \chi_{\Gamma_{c}}\right)\left(\sigma_{1}\right) E_{2, b}\left(S_{b}^{(2)} \chi_{\Gamma_{c}}\right)\left(\sigma_{2}\right)
\end{gathered}
$$

Now if, as it is done in many imprecise probabilistic models of the EPR experiment, one neglects the conditioning factor $1 / P\left(\Gamma_{c}\right)$, one would be lead to consider, instead of the correct integral (??), the integral

$$
\iint p_{S}\left(\sigma_{1}, \sigma_{2}\right) d \sigma_{1} d \sigma_{2} E_{1, a}\left(\hat{S}_{a}^{(1)}\right)\left(\sigma_{1}\right) E_{2, b}\left(\hat{S}_{b}^{(2)}\right)\left(\sigma_{2}\right)
$$


$\left(\hat{S}_{x}^{(j)}=S_{x}^{(j)} \chi_{\Gamma_{c}}\right)$. To this integral one can easily apply the considerations of Section (7) below and deduce the Bell inequality

$$
\left|\left\langle S_{a}^{(1)} S_{b}^{(2)}\right\rangle_{0}-\left\langle S_{c}^{(1)} S_{b}^{(2)}\right\rangle_{0}\right|-\left\langle S_{a}^{(1)} S_{c}^{(2)}\right\rangle_{0} \leq 1
$$

where $\langle\cdot\rangle_{0}$ means that the correlations are computed with the incorrect integral (??). However, if the correlations are computed with the correct integral (??) one has to multiply both sides of (??) by $1 / P\left(\Gamma_{c}\right)$ which leads to

$$
\left|\left\langle S_{a}^{(1)} S_{b}^{(2)}\right\rangle-\left\langle S_{c}^{(1)} S_{b}^{(2)}\right\rangle\right|-\left\langle S_{a}^{(1)} S_{b}^{(2)}\right\rangle \leq \frac{1}{P\left(\Gamma_{c}\right)}
$$

Now, in the notation (??), if we specify our system by the requirements that $p_{S}\left(\sigma_{1}, \sigma_{2}\right) d \sigma_{1} d \sigma_{2}$ is given by (??) and:

$$
\begin{gathered}
P\left(\Gamma_{c}\right)=(2 \pi)^{-1} \\
E_{1, a}\left(S_{a}^{(1)} \chi_{\Gamma_{c}}\right)\left(\sigma_{1}\right)=\int_{0}^{2 \pi} \frac{\left|\cos \left(\sigma_{1}-a\right)\right|}{4} S_{a}^{(1)}\left(\sigma_{1}, \lambda_{1}\right) \tilde{p}_{1, a}\left(\sigma_{1}, \lambda_{1}\right) d \lambda_{1} \\
E_{2, b}\left(S_{b}^{(2)} \chi_{\Gamma_{c}}\right)\left(\sigma_{2}\right)=\int_{0}^{2 \pi} S_{b}^{(2)}\left(\sigma_{2}, \lambda_{2}\right) \tilde{p}_{2, b}\left(\sigma_{2}, \lambda_{2}\right) d \lambda_{2}
\end{gathered}
$$

then we find the expression (??).

The choice we made in our experiment is very special. Formula (??) suggests that, by appropriately constructing deterministic dynamical systems, one can make the probability $P\left(\Gamma_{c}\right)$ arbitrarily small, hence the bound in (??) arbitrarily high.

\section{Conditioning on coincidences: direct simulation}

In the present section we describe a technique to simulate directly the conditional probabilities introduced in section (??). In this way the multiplication and division by $2 \pi$ comes out from the statistical countings themselves and the singlet condition is verified at each step of the simulation.

In the idealized dynamical system considered in our experiment we consider only two time instants 0 (initial) and 1 (final) so, in our case, a "trajectory" consists of a single jump. We do not describe the space-time details of the trajectory because we are only interested in distinguishing 2 cases:

- at time 1 the particle is in the apparatus (and in this case it is detected with certainty)

- at time 1 the particle is not in the apparatus (and in this case it makes no sense to speak of detection)

Thus our "configuration space" for the single particle will be made of 3 points: $s$ (source), 1 (inside apparatus), 0 (outside apparatus). Since at time 0 the "position" of both particles is always $s$, because of the chameleon effect, the position $q_{j, 1}$ of particle $j=(1,2)$ at time 1 will depend on the polarization $a_{j}$, on the initial state $\sigma$ and on the state $\lambda_{j}$ of the apparatus $M_{j}(j=1,2)$ :

$$
q_{j, 1}=q_{j, 1}\left(a_{j}, \sigma, \lambda_{j}\right) \quad ; \quad j=1,2,
$$

The local, deterministic dynamical law of this dependence is the one described in Section (5.4). 
There is no conceptual difficulty to include in our model the consideration of the spacetime trajectory of the particle. This surely would improve the present model, however the main conclusion of our experiment, i.e. the reproducibility of the EPR correlations by a classical, deterministic, local dynamical system, will not change.

\subsection{Description of the experiment}

1. Let $N \leq N_{\text {tot }}$ be natural integers and let

$$
\left\{\sigma_{j}: j=1, \ldots, N\right\}
$$

be the sequence of numbers

$$
\left\{\sigma_{j}:=(2 \pi / N) \times j: j=1, \ldots, N\right\}
$$

We have checked in several experiments that any pseudo-random sequence in $[0,2 \pi]$ with good equidistribution properties will lead to the same result. This fact is reflected in the option $D$ (deterministic) or $R$ (random) that has been inserted in the program of the experiment. Let $N\left(\sigma_{j}\right)(j=1, \ldots, N)$ denote a sequence of natural integers such that

$$
\sum_{j=1}^{N} N\left(\sigma_{j}\right)=N_{t o t}
$$

2. For each $j$ from 1 to $N$, repeat the following 3 operations (a), (b), (c), $N\left(\sigma_{j}\right)$ times

(a) The central computer sends $\sigma_{j}$ to the computers 1 and 2 .

(b) Computer 1 computes the position of particle 1 as described in section 5.4 below and sends back $S_{a}^{(1)}\left(\sigma_{j}\right)(=1$ or -1$)$ if the particle is inside the apparatus. It sends back $\emptyset$ (empty) if the particle is outside the apparatus. Similarly Computer 2 does the same thing. The dynamics is such that $S_{a}^{(1)}\left(\sigma_{j}\right)$ is sent back with probability $p_{1, a}\left(\sigma_{j}\right)$ and $S_{b}^{(2)}\left(\sigma_{j}\right)$ is sent back with probability $p_{2, b}\left(\sigma_{j}\right)$ where $p_{1, a}, p_{2, b}$ are sufficiently regular probability densities (say piecewise smooth with a finite number of discontinuities in $[0,2 \pi]$ (See sec.??)). This corresponds in the real experiments, to labeling the local detection time of the photon. When both computers send back a value \pm 1 , then we say that a coincidence occurs.

(c) Only in case of a coincidence, i.e. when the central computer receives the value \pm 1 from both computers, the central computer computes the "correlation product" $S_{a}^{(1)}\left(\sigma_{j}\right) S_{b}^{(2)}\left(\sigma_{j}\right)$

3. The central computer computes the correlation as

$$
\frac{\text { Sum of all correlation products }}{\text { The total number of coincidences }} \text {. }
$$

\subsection{Computation of the correlations}

Introducing

$$
p\left(\sigma_{j}\right)=\frac{N\left(\sigma_{j}\right)}{N_{\text {tot }}}
$$


the expected number of coincidences $\mathcal{N}_{\text {coincidences }}$ and the sum of all correlation products $\mathcal{S}_{\text {correlations }}$ become respectively

$$
\begin{aligned}
\mathcal{N}_{\text {coincidences }} & =\sum_{j=1}^{N} N\left(\sigma_{j}\right) p_{1, a}\left(\sigma_{j}\right) p_{2, b}\left(\sigma_{j}\right)=N_{\text {tot }} \sum_{j=1}^{N} p\left(\sigma_{j}\right) p_{1, a}\left(\sigma_{j}\right) p_{2, b}\left(\sigma_{j}\right) \\
\mathcal{S}_{\text {correlations }} & =\sum_{j=1}^{N} N\left(\sigma_{j}\right) p_{1, a}\left(\sigma_{j}\right) p_{2, b}\left(\sigma_{j}\right) S_{1}^{(a)}\left(\sigma_{j}\right) S_{2}^{(b)}\left(\sigma_{j}\right) \\
& =N_{\text {tot }} \sum_{j=1}^{N} p\left(\sigma_{j}\right) p_{1, a}\left(\sigma_{j}\right) p_{2, b}\left(\sigma_{j}\right) S_{1}^{(a)}\left(\sigma_{j}\right) S_{2}^{(b)}\left(\sigma_{j}\right)
\end{aligned}
$$

Thus the correlation defined by (??) is

$$
\frac{\mathcal{S}_{\text {correlations }}}{\mathcal{N}_{\text {coincidences }}}=\frac{\sum_{j=1}^{N} p\left(\sigma_{j}\right) p_{1, a}\left(\sigma_{j}\right) p_{2, b}\left(\sigma_{j}\right) S_{1}^{(a)}\left(\sigma_{j}\right) S_{2}^{(b)}\left(\sigma_{j}\right)}{\sum_{j=1}^{N} p\left(\sigma_{j}\right) p_{1, a}\left(\sigma_{j}\right) p_{2, b}\left(\sigma_{j}\right)}
$$

and therefore

$$
\frac{\mathcal{S}_{\text {correlations }}}{\mathcal{N}_{\text {coincidences }}} \rightarrow \frac{\int_{0}^{2 \pi} d \sigma p(\sigma) p_{1, a}(\sigma) p_{2, b}(\sigma) S_{1}^{(a)}(\sigma) S_{2}^{(b)}(\sigma)}{\int_{0}^{2 \pi} d \sigma p(\sigma) p_{1, a}(\sigma) p_{2, b}(\sigma)}
$$

where $p(\sigma)$ is a probability density with properties analogue to $p_{1, a}$ and $p_{2, b}$.

\subsection{Realization of the EPR correlation}

With the choices:

$$
\begin{aligned}
& p(\sigma)=\frac{1}{2 \pi} \quad, \quad p_{1, a}(\sigma)=\frac{1}{4}|\cos (\sigma-a)| \quad, \quad p_{2, b}(\sigma)=1 \\
& S_{1}^{(a)}(\sigma)=\operatorname{sgn}(\cos (\sigma-a)), \quad S_{2}^{(b)}(\sigma)=-\operatorname{sgn}(\cos (\sigma-b))
\end{aligned}
$$

we obtain

$$
\begin{gathered}
\text { numerator of }(? ?)=-\frac{1}{2 \pi} \cos (a-b) \\
\text { denominator of }(? ?)=\frac{1}{2 \pi}
\end{gathered}
$$

Therefore for large $N$ the correlation (??) is well approximated by

$$
-\cos (a-b)
$$

which is exactly the EPR correlation. We underline that, as shown by (??), even if the mechanism of coincidences depends on the setting of the apparatus, the expected number of coincidences is independent of it, in agreement with the experimental result quoted in the introduction. 


\subsection{Computation of the position}

With the choices of section (??), $p_{2, b}(\sigma)$ is trivial $(=1)$ and this means that computer 2 always sends back $S_{2}^{(b)}(\sigma)$ to the central computer. On the other hand, computer 1 associates the label 1 to $S_{1}^{(a)}(\sigma)$ in the following way,

1. For each input $\sigma_{j}$, generate a new random variable $\lambda_{1}$ with a probability distribution $P_{01}\left(\lambda_{1}\right)=\chi_{[0,1]}$.

2. When $\lambda_{1}$ is such that $0 \leq \lambda_{1} \leq p_{1, a}(\sigma)$ computer 1 concludes that the particle is inside sends the value $S_{1}^{(a)}\left(\sigma_{j}\right)$, otherwise it sends $\emptyset$.

\section{Difference between coincidences and efficiency of the detectors}

As already emphasized in the introduction of the present paper, the difference between conditioning on coincidences and efficiency detectors is a principle one. Moreover, in many experimental situations, the total number of pairs emitted by the source is in principle unboservable. For example in all EPR type experiments with photons, the source of entangled pairs has a finite size hence the probability that one or both photons of some pair is reabsorbed by the source itself is nonzero. In all these cases the statistical counting is conditioned on the simultaneously detected pairs. Thus, whenever we want to estimate statistically equal time correlations of the form

$$
\left\langle S_{a}^{(1)}(t) S_{b}^{(2)}(t)\right\rangle
$$

we must be aware that in general they will be correlations conditioned on coincidences.

In the loophole argument, the following fact has been noted: Einstein's local realism can be consistent with the experimental data when the excessive correlations might be possessed only by a fraction of the coincident pairs actually detected. In other words, an high efficiency of the detectors is required to exclude the loophole.

Conditioning on coincidences has nothing to do with these arguments on the efficiency of the detectors because, as clearly explained in these arguments, the efficiency is calibrated with the ratio of the number of particles detected by the detector with polarizer and without polarizer, while the core of the conditioning argument is that the number of detected particles without polarizer may not be the total number of particles emitted by the source. This number is usually unobservable in a real experiment even if one postulates $100 \%$ efficiency in the detection. In the real experiments (e.g. [?, ?, ?]), before taking the coincidences we cannot speak of the total number of particles satisfying the singlet condition.

Thus conditioning on coincidences has nothing to do with the loophole argument. In this section we discuss the mathematical difference between the two points of view and we show how this can be experimentally verified. At the end of this section we also explain the role of the dynamics (chameleon effect) in establishing the coincidences.

In order to motivate and describe more precisely the difference between this type of correlations and the unconditional ones, suppose we have two particles $(1,2)$ with the same state space $S$ and let

$$
\left(\sigma_{1, t}\right) ; \quad\left(\sigma_{2, t}\right) ; \quad\left(\sigma_{t}\right)=\left(\sigma_{1, t}, \sigma_{2, t}\right) ; \quad t \in \mathbb{R}
$$

be the stochastic processes describing the time evolution of these particles in the state space (deterministic processes are a particular case). 
Suppose that the process (??) is stationary and ergodic and that we want to measure experimentally the equal time correlations of two functions $S_{a}^{(1)}, S_{b}^{(2)}$ of these particles.

If we do $N$ independent measurements in our laboratory at times $t_{1}, \ldots, t_{N}$ finding the results $\sigma_{1}, \ldots, \sigma_{N}$ respectively, can we conclude that

$$
\frac{1}{N} \sum_{j=1}^{N} S_{a}^{(1)}\left(\sigma_{j}\right) S_{b}^{(2)}\left(\sigma_{j}\right) \sim E\left(S_{a}^{(1)}(t) S_{b}^{(2)}(t)\right)=\left\langle S_{a}^{(1)}(t) S_{b}^{(2)}(t)\right\rangle
$$

as a naive application of the ergodic theorem would suggest? The answer is clearly: no. In fact the states $\sigma_{1, t}, \sigma_{2, t}$ will in general depend on many parameters such as position, momentum, spin, polarization,...

$$
\sigma_{1, t}=\sigma_{1, t}\left(q_{1, t}, p_{1, t}, \ldots\right)
$$

and, by our assumption, the effective measurements are done in the bounded space regions $A_{1}$ and $B_{2}$. This means that we are not counting all the emitted particles but only those which happened to be in the same time in the regions $A_{1}$ and $B_{2}$ (recall that we are postulating $100 \%$ efficient detectors). This is precisely what characterizes conditional probability. Therefore the correct conclusion is not (??) but

$$
\frac{1}{N} \sum_{j=1}^{N} S_{a}^{(1)}\left(\sigma_{j}\right) S_{b}^{(2)}\left(\sigma_{j}\right) \sim E\left(S_{a}^{(1)}\left(\sigma_{t}\right) S_{b}^{(2)}\left(\sigma_{t}\right) \mid q_{1}(t) \in A_{1} ; q_{2}(t) \in B_{2}\right)
$$

Thus, if we say that a coincidence occurs if, for some $t$ one has both $q_{1}(t) \in A_{1}$ and $q_{2}(t) \in B_{2}$ and we denote

$$
\Gamma_{c}\left(\sigma_{t}\right):=\left[q_{1}(t) \in A_{1} \quad \text { and } \quad q_{2}(t) \in B_{2}\right]
$$

the corresponding event (notice that both $q_{1}(t)$ and $q_{2}(t)$ are functions of $\sigma_{t}$ ), then we can rewrite (??) in the form

$$
\begin{gathered}
\frac{1}{N} \sum_{j=1}^{N} S_{a}^{(1)}\left(\sigma_{j}\right) S_{b}^{(2)}\left(\sigma_{j}\right) \sim E\left(S_{a}^{(1)}\left(\sigma_{1, t}\right) S_{b}^{(2)}\left(\sigma_{2, t}\right) \mid \Gamma_{c}\left(\sigma_{t}\right)\right)= \\
=\frac{E\left(S_{a}^{(1)}\left(\sigma_{t}\right) S_{b}^{(2)}\left(\sigma_{t}\right) \chi_{\Gamma_{c}(\sigma(t))}\right)}{P\left(\Gamma_{c}(\sigma(t))\right)}
\end{gathered}
$$

Because of the stationarity of the process, the right hand side of (??) can be written

$$
\frac{E\left(S_{a}^{(1)} S_{b}^{(2)} \chi_{\Gamma_{c}}\right)}{P\left(\Gamma_{c}\right)}
$$

The conclusion (??) would be justified only if one could prove that the number $N$, appearing in it, is the total number of emitted pairs which, in many sitautions, is unboservable in principle.

As shown by the considerations above, the class of stochastic processes such that there exist two regions $A_{1}$ and $B_{2}$ for which this is the case, is very special (although surely nonempty).

In conclusion, let us consider a simple example, concerning a single polarizer which may help clarifying the conceptual and experimental difference between the efficiency and the coincidence problems. 
Suppose that a detector is $100 \%$ efficient. Then, if a source emits 100 photons, all photons are detected in absence of polarizer. Suppose moreover that, when the polarizer is inserted, only 90 photons and not 100 are detected. Therefore, if as done in [?], the efficiency is calibrated with the ratio of the number of particles detected by the detector with polarizer and without polarizer, we should conclude that our polarizer is $90 \%$ efficient.

However, if the loss of these 10 photons is due to the chameleon effect, then by repeating many times the experiment (and postulating a situation of stationarity of the source) one should always detect 90 photons.

On the contrary, if the loss of photons is due to accidental causes, then the number of detected photons should fluctuate and an analysis of these fluctuations should, in principle, allow to distinguish between an $100 \%$ efficient detector in presence of the chameleon effect and an $100 \%$ efficient detector in presence of a $90 \%$ efficient polarizer.

In real physical situations the two effects are most likely combined and their distinction, although clear in principle, might be a very hard challenge both for theoreticians and experimentalists. However we are convinced that a satisfactory theory of measurement should take into account both these effects.

\section{Why contextuality is not enough}

The following theorem shows that the contextuality argument alone, advocated by several authors[?, ?] is not enough to rule out the application of Bell's inequality. In fact this theorem implies that there is a large class of contextual hidden variable theories, i.e. in which the initial distribution depends on the global setting $a, b$ of the far away apparata, which satisfy the Bell inequality and which therefore cannot reproduce the singlet correlations. This shows in particular that to achieve such a violation

(i) dynamical considerations (chameleon effect) are necessary

(ii) in the local decomposition of our probability measure (??) the non normalization condition (??) of the local measures $p_{1, a}, p_{2, b}$, i.e. the fact that they are not conditional probabilities in the sense of Remark (1) of Section (2), is a necessary condition for the above mentioned violation.

Theorem 1 Let $\mathcal{A}_{1}, \mathcal{A}_{2}$ (system observables) and $\mathcal{A}_{M_{1}}, \mathcal{A}_{M_{2}}$ (apparatus observables) be commutative *-algebras and, for any such algebra $\mathcal{A}$ denote $\mathcal{S}(\mathcal{A})$ the set of its states. Suppose that, for any pair of vectors in the unit sphere $S^{(2)}$ in 3 -dimensional space:

(i) the initial state of the system $\left(1,2, M_{1}, M_{2}\right)$ has the form

$$
\psi_{a, b}=\psi_{1,2} \circ\left(E_{1, a} \otimes E_{2, b}\right)
$$

where $\psi_{1,2} \in \mathcal{S}\left(\mathcal{A}_{1} \otimes \mathcal{A}_{2}\right)$ and

$$
E_{j, x}: \mathcal{A}_{j} \otimes \mathcal{A}_{M_{j}} \rightarrow \mathcal{A}_{j} \quad ; \quad j=1,2 ; x \in S^{(2)}
$$

are conditional expectations.

(ii) For each $j=1,2$ and $x \in S^{(2)}$ it is given a self adjoint element $S_{x}^{(j)}$ in $\mathcal{A}_{j}$ with spectrum in the interval $[-1,1]$ and a positive normalized map $T_{j, x}: \mathcal{A}_{j} \rightarrow \mathcal{A}_{j}\left(T_{j, x}(1)=1\right.$. Then the pair correlations

$$
\left\langle S_{a}^{(1)} S_{b}^{(2)}\right\rangle=\psi_{a, b}\left(T_{1, a}\left(S_{a}^{(1)}\right) T_{2, b}\left(S_{b}^{(2)}\right)\right)=\psi_{1,2}\left(E_{1, a}\left(T_{1, a}\left(S_{a}^{(1)}\right)\right) E_{2, b}\left(T_{2, b}\left(S_{b}^{(2)}\right)\right)\right)
$$

cannot be the EPR ones. 
Proof. By contradiction. Consider the 4 random variables in $\mathcal{A}_{1} \otimes \mathcal{A}_{2}$ :

$$
E_{1, a}\left(T_{1, a}\left(S_{a}^{(1)}\right)\right), E_{2, b}\left(T_{2, b}\left(S_{b}^{(2)}\right)\right), E_{1, c}\left(T_{1, c}\left(S_{c}^{(1)}\right)\right), E_{2, c}\left(T_{2, c}\left(S_{c}^{(2)}\right)\right)
$$

The positivity and normalization of the dynamics implies that

$$
-1 \leq T_{j, x}\left(S_{x}^{(j)}\right) \leq+1 \quad ; \quad j=1,2 ; x \in S^{(2)}
$$

hence the same inequalities hold for the random variables (??). Moreover, if the correlations (??) are equal to $-a \cdot b$ then, for any $c \in S^{(2)}$,

$$
E_{1, c}\left(T_{1, c}\left(S_{c}^{(1)}\right)\right)=-E_{2, c}\left(T_{2, c}\left(S_{c}^{(2)}\right)\right) ; \quad \psi_{1,2}-\text { a.e. }
$$

(cf. Lemma (14.1) in [?]) and from this the Bell's inequality follows.

Corollary 1 If the initial state of the system has the form

$$
\psi_{12} \otimes \psi_{1, a} \otimes \psi_{2, b}
$$

with $\psi_{1,2} \in \mathcal{S}\left(\mathcal{A}_{1} \otimes \mathcal{A}_{2}\right), \psi_{j, x} \in \mathcal{S}\left(\mathcal{A}_{M_{j}}\right), j=1,2, \forall x$ and if the dynamics satisfy (??) then the pair correlations

$$
\left\langle S_{a}^{(1)} S_{b}^{(2)}\right\rangle=\left(\psi_{12} \otimes \psi_{1, a} \otimes \psi_{2, b}\right)\left(T_{1, a} \otimes T_{2, b}\right)\left(S_{a}^{(1)} \otimes S_{b}^{(2)}\right)
$$

cannot be the EPR ones.

Proof. Apply Theorem (??) to the case

$$
E_{j, x}\left(a_{j} \otimes a_{M_{1}}\right)=a_{j} \psi_{j, x}\left(a_{M_{j}}\right) ; \quad j=1,2 ; \quad a_{j} \in \mathcal{A}_{j} ; \quad a_{M_{j}} \in \mathcal{A}_{M_{j}}
$$

\section{Conclusions}

The main point of the present experiment is not, as already emphasized in the introduction, to build a hidden variable theory for the EPR experiment: the main problem with hidden vaiable theories is not so much their existence as their non uniqueness. This implies that an experimental discrimination between any two such theories would be impossible due to the Heisenberg principle.

The main idea of the "chameleon philosophy" can be summarized in the words of A. Tartaglia [?]: "... In conclusion quantum measurements and the story of the violation of the Bell or similar inequalities tell us that the objects of the quantum world are not like boxes containing spin, polarization vector etc. like buttons, pins, pearls and the like, but like programmed machines capable of different behaviours according to the physical conditions locally triggering them. ..."

We have a great admiration for the extraordinary experiments that have been done and continue to be done in this field and their value is in by no way belittled by the present experiment.

Concerning the detection loophole argument we believe that the distinction between chameleon effect (which has a principle nature) and random errors (which are contingent) should stimulate a deeper discussion of the very notion of "efficiency of an apparatus", leading to a distinction, in the role played by the interaction system-apparatus in the determination 
of the observed statistics, between the theoretically describable, at least in principle, interaction between system and apparatus and the accidental errors such as spurious photons, imprecisions of clocks, imprecise determination of the initial state, generic malfunctioning, ... . The existing models, based on the efficiency argument, can be refined without much effort by including a distinction between these two types of inefficiencies.

Finally, as far as quantum cryptography is concerned, we emphasize the possible use of dynamical systems, such as the one discussed in the present paper, both as a benchmark for truly quantum algorithms and as a stimulus for potentially fruitful investigations on hybrid algorithms, i.e. combining classical and quantum features, with a potential impact on lowering the costs and increasing the performance of the usual quantum cryptographic algorithms.

\section{Acknowledgment}

The autors are grateful to Richard Gill whose fierce opposition to their ideas surely contributed to improve the presentation of the present paper.

\section{References}

[1] Luigi Accardi, Massimo Regoli, Locality and Bell's inequality. Preprint Volterra, N. 427 (2000), quant-ph/0007005.

[2] Luigi Accardi, Massimo Regoli, The EPR correlations and the chameleon effect, discussed during the "Japan-Italy Joint workshop on Quantum open systems and quantum measurement", Waseda University, 27-29 September 2001, quant-ph/0110086, Volterra preprint No. 487 (2001).

[3] Bell J.S, "On the Einstein Podolsky Rosen Paradox", Physics 1 no. 3 (1964) 195-200.

[4] Accardi L., Topics in quantum probability, Phys. Rep. 77 (1981) 169-192

[5] L. Accardi, Einstein-Bohr: one all, in: The interpretation of quantum theory: where do we stand? Acta Enciclopedica, Istituto dell'Enciclopedia Italiana (1994) 95-115, Volterra preprint No. 174 (1993).

[6] Angelo Tartaglia: Is the EPR paradox a paradox? Eur. J. Phys., 19 (1998) 307

[7] Phillip M. Pearle, Phys. Rev. D 2, 1418 (1970),

[8] J. F. Clauser and M. A. Horne, Phys. Rev. D 10, 526 (1974),

[9] J. F. Clauser, M. A. Horne, A. Shimony, and R. A. Holt, Phys. Rev. Lett. 23880 (1969),

[10] T. K. Lo and A. Shimony, Phys. Rev. A 233003 (1981),

[11] Anupam Garg and N. D. Mermin, Phys. Rev. D 353831 (1987).

[12] Gisin N., Gisin B., A local hidden variable model of quantum correlation exploiting the detection loophole, Phys. Lett. A 260 (1999) 323-327, Quant-ph/9905018 6 may 1999, version of April, 22 (2001)

[13] Laszlo E. Szabo, Arthur Fine, A local hidden variable theory for the GHZ experiment, quant-ph/0007102 
[14] G. Brassard, R. Cleve and A. Tapp, The cost of exactly simulating quantum entanglement with classical communication, quant-ph/9901035

[15] M. Steiner, towards quantifying non-local information transfer: finite-bit non-locality, quant-ph/9902014

[16] Gregor Weih, Thomas Jennewein, Cristoph Simon, Harald Weinfurter and Anton Zeillinger: Violation of Bell's inequalitiy under strict Einstein locality conditions, Phys. Rev. Lett. 81 (1998) 5039-5043

[17] Sergienko A.V., Atature M., Walton Z., Jaeger G., Saleh B.E.A., Teich M.C., Quantum cryptography using femtosecond-pulsed parametric down-conversion, Phys. Rev. A60, (1999) R2622.

[18] Alain Aspect, Philippe Grangier, and Gerard Roger, Phys. Rev. Lett. 49, 91 (1982).

[19] de Muynck W.M., De Baere W., Martens H.: Interpretations of quantum mechanics, joint measurement of incompatible observables, and counterfactual definiteness, Found. of Phys. 24, (1994) 1589-1663.

[20] A.Yu. Khrennikov: Non-Archimedean analysis: quantum paradoxes, dynamical systems and biological models. Kluwer Acad.Publ., Dordreht (1997). 